\title{
Regulatory Inspection of Clinical Trials
}

\section{Hitt Sharma*}

Serum Institute of India Ltd, 212/ 2, Hadapsar, Pune - 411028, India

The International Conference on Harmonization defines "inspection" as "The act by a regulatory authority (ies) of conducting an official review of documents, facilities, records, and any other resources that are deemed by the authority (ies) to be related to the clinical trial and that may be located at the site of the trial, at the sponsor's and/or contract research organization's (CRO's) facilities, or at other establishments deemed appropriate by the regulatory authority (ies)".

Quality of trial processes and generated data, as well as compliance with all applicable international and local regulations, should be always regarded as a continuous process. So, all participants of a clinical trial process must expect and be always prepared for any kind of inspections and/or audits, notwithstanding presence or absence of the foreseen triggers (high/ low enrollment and dropout rates, unusual AE/ SAE number, study risk profile, etc.) [1].

\section{What to Expect During a Routine Inspection?}

The inspections consist of site visits to the organization and, where appropriate, selected clinical investigator sites. The inspection will start with an opening meeting where the lead inspector will outline the scope and purpose of the inspection, confirm the inspection plan and introduce the inspector(s). During the inspection, the inspectors will request additional documentation and the organization should be prepared to provide this promptly. Interviews with relevant personnel, generally as per the inspection plan, are conducted. Time is built into the inspection for the review of documentation, for example the TMF for the selected clinical trials. The inspector may visit any facilities involved in clinical trials, for example data management units, archives, pharmacy and these visits may be pre-arranged by the inspector for logistical reasons or decided upon during the inspection. During the inspection, the inspectors reserve the right to change the inspection plan, for example additional interviews or time may be required dependent upon inspection findings. At the end of the inspection there will be a closing meeting where the findings are reported verbally to the organization, next steps given regarding any further sites to be inspected and the time scales for the report [2].

\section{Common Findings}

Protocol noncompliance, inadequate/inaccurate records, inadequate drug accountability, informed consent issues, and adverse event reporting are some of the most common findings observed during inspections. In this regard, a warning letter is issued to the clinical investigators by the National Regulatory Authority (NRA). The warning letter clearly demonstrates that GCP violations are unacceptable; in case they occur, immediate and effective actions are expected; principal investigator is responsible for site's compliance to GCP norms and will be held accountable for noncompliance, lack of or delayed action plans to resolve and prevent the issues may result in further actions by NRA. In addition, the sponsor may be criticized for not having identified the issues and not taking actions, such as holding further patient enrolment till the issues are resolved or have improved. In fact, the best strategy for clinical research associates to prepare themselves and their sites for any type of inspections/audits is to strictly and constantly adhere to standard operating procedures and all applicable regulatory guidelines. If you want something to be fulfilled, you must control and measure it. If it is not being done underway, there is no sense to do major preparations within a week or two before an audit - it will be evident for an experienced auditor/inspector after all.

\section{Scope and Preparations}

The scope of preparations depends, of course, on the areas that are usually checked during the inspections (and audits) and on the specific SOPs of the Sponsor/CRO. In the case of the regulatory inspection the following aspects are usually reviewed and verified, as specified by $[3,4]$ :

- Site's procedures and study conduct and whether these correspond to what was stated in the clinical trial application

- Adequacy of communication with the IRB, including the initial submission document, adverse event reporting, and progress reports.

- Appropriateness of the informed consent process

- Whether submitted data reflect actually occurred events

- Whether study has been conducted according to GCP and other regulatory requirements

- Sponsor's/ CRO's/ Site's SOPs

- Local guidelines

- Compliance with the study protocol and documentation that each deviation/amendment received IRB and sponsor approval

- Results of previous inspections, if any

- Completeness of accountability documentation for the receipt, storage, administration and return of test article (drug, device, etc.);

- Adequate monitoring of the site and communication with the sponsor.

Generally, it is better to ensure that site staff has all documents ready and in place and will be present during the inspection. I would also instruct them not to devise anything and tell the truth. Even if something is missing it is better to document the list of pending/ missing documents then to re-write lost logs / charts (whatever) over the period of time before the inspection [5].

Some tips that may help to ensure a smooth conduct of an inspection are - to prepare for inspection from day 1, i.e., always be Good Clinical Practices and regulations compliant; know your protocol and study requirements well; organize documentation well and provide accurate, complete, final version of documents requested; have key

Corresponding author: Hitt Sharma, Additional Director (Medical Affairs), Serum Institute of India Ltd, 212/ 2, Hadapsar, Pune - 411028, India, Tel: + 91-2026602451; Fax: +91-20-26998146; Email: drhjs@seruminstitute.com

Received March 12, 2012; Accepted March 14, 2012; Published March 19, 2012

Citation: Sharma H (2012) Regulatory Inspection of Clinical Trials. Pharmaceut Reg Affairs 1:e103. doi:10.4172/2167-7689.1000e103

Copyright: @ 2012 Sharma H. This is an open-access article distributed under the terms of the Creative Commons Attribution License, which permits unrestricted use, distribution, and reproduction in any medium, provided the original author and source are credited. 
personnel/ subject matter experts available to respond to questions; answer questions only in your area of responsibility; request clarification on any question or issue that is not clear, answer only the question asked and be upfront if you don't know the answer, if follow-up is needed in order to respond to question, follow-up immediately; provide privacy to inspection team, but periodically check on progress and ask if clarification is needed; keep track of requested documents and provide them at the earliest; prepare overview of study conduct/ roles and responsibilities of staff; maintain respectful, professional, and cooperative demeanor.

\section{Future}

With an increase in number of clinical trials happening around the globe, it is clear that that enforcement will be swift, aggressive, and effective. Companies are expected to strictly adhere to legal requirements designed to protect product quality and integrity. All parties involved in the drug lifecycle must recognize that they have an ethical liability [6]. It is important that local supervision in every country is supported and strengthened through capacity building, networking, information exchange, and by taking advantage of opportunities for joint or observed inspections. The sponsor is responsible for implementing a system of quality assurance in order to ensure that the trial is performed and the data are generated, recorded, and reported in compliance with the protocol, Good Clinical Practice, and national regulations. All clinical trial sites, data and documents must be available for verification. All observations and findings should be verifiable in order to ensure the credibility of data and to assure that the conclusions presented are derived correctly from the raw data. The verification processes must be specified and scientifically justified. Statistically controlled sampling may be used to verify data in a trial. Quality control procedures must be applied to each stage of data handling to ensure that all data are reliable and have been processed correctly. The sponsor, investigational sites, facilities and laboratories, and all relevant data (including raw data) and documentation and reports concerning the data (including subject files) must be available for an audit and for inspection by relevant health authorities. Such arrangements will provide adequate assurance that the study is planned and conducted according to generally accepted scientific principles and Good Clinical Practice.

\section{Conclusion}

Drug development is being increasingly globalized and an increased number of patients enrolled in studies submitted as part of applications come from all over the world.

However, because of the steep increase in research activity in the country, inexperienced sites, and more stakeholders, increased efforts are required to ensure continuous quality and compliance. It is expected that clinical trials are conducted according to the ethical and data quality requirements. Inspectors are collaborating and sharing work through joint inspections with an aim to ensure that the trials meet the standards for good clinical practice (GCP). This includes attention to trial subject's well being, safety and rights as well as good data quality. Health authorities have also made clear that enforcement will be increased and be swift, aggressive, and effective.

\section{References}

1. Valania M (2006) Quality Control and Assurance in Clinical Research - A system of checks and examinations that helps ensure the quality of clinical trials. Applied Clinical Trials.

2. Medicine and Healthcare products Regulatory Agency (2010) Good Clinical Practice: The inspection process

3. Holford S (2003) Keeping an eye on the incubators. PharmaFocus.

4. Kurdziel TJ (2008) Regulatory Authority Inspections - Lessons Learned, New Zealand Clinical Research Conference, August, Auckland, New Zealand.

5. UK Medical Research Council Clinical Trials Toolkit (2006) How to prepare for an inspection for Good Clinical Practice by the Medicines and Healthcare products Regulatory Agency (MHRA): a guide for NHS organisations that sponsor or host clinical trials of medicinal product.

6. Marwah R, Van de Voorde K, Parchman J (2010) Good clinical practice regulatory inspections: Lessons for Indian investigator sites. Perspect Clin Res 1:151-155. 